\title{
The Role of Endoscopic Ultrasound in the Diagnosis of Cystic Lesions of the Pancreas
}

\author{
Philippe Lévya,b,c Vinciane Rebours ${ }^{a, b, c}$ \\ ${ }^{a}$ Department of Gastroenterology and Pancreatology, Pôle des Maladies de I'Appareil Digestif, DHU Unity, Hôpital Beaujon, \\ Faculté Denis Diderot, AP-HP, Clichy, France; \\ ${ }^{b}$ Centre de référence des maladies rares du pancréas (PAncreatic RAre DISeases), Hôpital Beaujon, Clichy, France; \\ ${ }^{c}$ Centre de référence européen des tumeurs neuroendocrines digestives et pancréatiques, Hôpital Beaujon, Clichy, France
}

\section{Keywords}

Serous cystic neoplasm - Mucinous cystic neoplasm . Intraductal papillary mucinous neoplasm .

Magnetic resonance imaging - Endoscopic ultrasound

\section{Summary}

A precise diagnosis of the nature of pancreatic cystic neoplasm (PCN) is crucial since it determines the patients in need of rapid surgical resection as well as those who can be followed up, and, accordingly, the frequency and modalities of surveillance. Endoscopic ultrasound (EUS) and especially fine needle aspiration (FNA) are invasive methods, with specific adverse events occurring in 2.7$5 \%$. Thus, they should only be used as a third-line tool in the absence of characteristic radiographic features on computed tomography (CT) scan and magnetic resonance imaging (MRI). The most difficult aspects of differential diagnosis are: intraductal papillary mucinous neoplasm (IPMN) versus chronic pancreatitis; unifocal IPMN versus serous cystic neoplasm (SCN); macrocystic SCN versus mucinous cystic neoplasm (MCN); cystic neuroendocrine tumors versus $\mathrm{MCN}$; solid serous cystadenoma versus neuroendocrine tumors versus small solid pseudopapillary tumors; pseudocyst versus MCN; low-grade, high-grade, or invasive IPMN. When classical radiological and EUS features are not conclusive, EUSFNA may be helpful by analyzing cytological, chemical, and/or molecular data. The addition of EUS-FNA to CT scan and MRI increased the overall accuracy for diagnosing PCN by 36 and 54\%, respectively. Analysis of molecular markers in pancreatic cyst fluid might increase the limited accuracy of EUS-FNA by using cytology and chemical and/or tumor marker analysis alone. Current evidence suggests that contrast-enhanced EUS ( $\mathrm{CH}$-EUS) is highly accurate for distinguishing non-neoplastic cysts from neoplastic cysts. CH-EUS might also be useful for distinguishing mural epithelial nodules from mucinous clots. Needle-based confocal laser endomicroscopy (nCLE) images a target tissue at a subcellular level of resolution, providing real-time in-vivo optical biopsy. nCLE is feasible during EUS-FNA and allows in-vivo diagnosis of PCN with high accuracy. In conclusion, EUS is a third-line tool in the diagnosis of PCN. Clinical context as well as careful evaluation of CT scan and magnetic resonance cholangiopancreatography images by specialized radiologists are crucial in the diagnosis process. Nowadays it is inconceivable to skip these steps.

(C) 2018 S. Karger GmbH, Freiburg

\section{Introduction}

Pancreatic cystic neoplasms (PCN) are more and more frequently diagnosed due to an increased utilization of imaging procedures like ultrasound, computed tomography (CT) scan, and magnetic resonance imaging (MRI). For instance, intraductal papillary mucinous neoplasms (IPMN) are found in 6.6\% in the general adult population and in $11.4 \%$ in those older than 60 years [1].

A precise diagnosis of the nature of PCN is crucial since it determines the patients in need of rapid surgical resection as well as those who can be followed up, and, accordingly, the frequency and modalities of surveillance. The 'perfect tool' should diagnose highgrade dysplasia, which is the best threshold to perform prophylactic pancreatectomy.

Knowledge of PCN has considerably increased since the 1990s. At that time, CT scan was not as accurate as today, MRI was just beginning, and magnetic resonance cholangiopancreatography

\section{KARGER}

(c) 2018 S. Karger GmbH, Freiburg 
(MRCP) did not exist at all. In parallel, endoscopic ultrasound (EUS) was developed. As a consequence, diagnosis and follow-up of PCN were mainly based on EUS and EUS-guided fine needle aspiration (EUS-FNA). During the following 20 years, the technical characteristics of CT scan and the development of MRCP have changed the diagnostic strategy.

EUS and especially FNA are considered as invasive methods due to the associated risks - although low - of general anesthesia, endoscopy, and FNA. Specific adverse events associated with EUS and EUS-FNA performed for PCN occur in 2.7-5\% [2].

Therefore, the first message is that a workup for PCN should include as a prerequisite both an excellent CT scan (thin slices $<2$ $\mathrm{mm}$ width; three phases (no iodine IV contrast, arterial and portal phases)) and MRCP (thick T2 slices centered on the main pancreatic duct at the head and body/tail level).

EUS should now be considered as a third-line tool in the absence of characteristic radiographic features. The goal of EUS \pm FNA is to refine diagnosis, to distinguish benign from premalignant lesions, and, for the latter group, to bring out signs that are considered as worrisome features or high-risk stigmata for malignant transformation.

All imaging techniques (radiological and endoscopic) are operator-dependent. As a consequence, interpretation of these data should be performed - or re-performed - by experienced gastroenterologists and radiologists for PCN.

\section{Morphological Features of Cystic Pancreatic Neoplasms}

EUS reports have to be very precise, describing all cystic lesions within the pancreas, pancreatic parenchyma, size and regularity of the main pancreatic duct, presence of mucus, mural nodules, and wall thickness. In practice, it is clear that this is not the case in a non-negligible number of patients.

The most difficult diagnostic task is to distinguish benign tumors from malignant or premalignant ones. The most difficult aspects of differential diagnosis are the following:

- IPMN versus chronic pancreatitis;

- unifocal IPMN versus serous cystic neoplasm (SCN);

- macrocystic SCN versus mucinous cystic neoplasm (MCN);

- cystic neuroendocrine tumors versus MCN;

- solid serous cystadenoma versus neuroendocrine tumors versus small solid pseudopapillary tumors;

- pseudocyst versus MCN;

- low-grade, high-grade, or invasive IPMN.

In the majority of cases, the clinical context and a careful interpretation of CT scan and MRCP are sufficient to reach a correct diagnosis. EUS is only warranted in the remaining cases.

Different EUS findings have been evaluated as diagnostic criteria for PCN. In all of the studies, however, the appropriateness of the radiological workup is not clear. The diagnostic accuracy of EUS imaging ranges from 40 to $96 \%$. One single prospective study demonstrated that the sensitivity (56\%), specificity (45\%), and ac- curacy (51\%) of EUS alone for differentiating MCN and IPMN from non-mucinous cysts were low [3]. For experienced endosonographers, the diagnosis by means of EUS of whether a cyst was neoplastic versus non-neoplastic was agreeable [4].

Some EUS features are predictive of types of cystic lesions:

- Parenchymal features suggestive of pancreatitis (calcifications, atrophy, inhomogeneous echo texture) associated with a cystic lesion indicates a pseudocyst with a sensitivity of $94 \%$ and a specificity of $85 \%$ [5].

- Multiple microcystic lesions (<3 mm) within a cystic lesion suggest an SCN with an accuracy of $92-96 \%$ [6]. This feature has to be searched for in all areas of a macrocystic lesion since its presence is predictive of the diagnosis of SCN [7]. This feature is not seen in MCN.

- Although EUS is probably the best imaging tool to reliably distinguish benign from malignant IPMN, the yield remains insufficient. A meta-analysis of 23 studies with 1,373 patients found that a mural nodule, main pancreatic duct dilation, thickened septal walls, and cyst size $>3 \mathrm{~cm}$ on radiological or EUS imaging were independent predictors of malignant branch-duct IPMN (BD-IPMN) [8]. In the recent revised international consensus guideline, EUS was recommended as a second-line tool in case of IPMN with worrisome features defined as pancreatitis, cyst size $>3 \mathrm{~cm}$, enhancing mural nodule $<5 \mathrm{~mm}$, thickened/enhancing cyst walls, main duct size of 5-9 $\mathrm{mm}$, abrupt change in caliber of pancreatic duct with distal pancreatic atrophy, lymphadenopathy, increased serum level of carbohydrate antigen (CA) 19-9, and a cyst growth rate $>5$ $\mathrm{mm} / 2$ years. On the other hand, a main pancreatic duct size $>$ $10 \mathrm{~mm}$ or the presence of an enhancing solid component on radiological imaging are considered as high-risk stigmata [9]. In the vast majority of cases, EUS is not warranted in order to decide on pancreatic resection.

- Distinguishing intracystic nodules is of paramount importance to stratify the risk of malignant evolution: EUS might help to differenciate epithelial (neoplastic) nodules from those that are mucinous (non-neoplastic) plugs. A recent blinded interobserver study found that the latter appear as a smooth, well-defined hyperechoic rim with a hypoechoic center compared with the surrounding parenchyma. On the contrary, true epithelial nodules have ill-defined borders and a hyperechoic center [10].

\section{Fine Needle Aspiration}

EUS-FNA has shown its usefulness in confirming a diagnosis of microcystic serous neoplasms; however, this practice is rarely necessary since imaging alone is often sufficient to reach a diagnosis of these tumors. When classical radiological and EUS features are not conclusive (once again, after a careful workup with pancreatologists), EUS-FNA may be helpful by analyzing cytological, chemical, and/or molecular data. Any solid component associated with a cystic lesion or regional lymph nodes can be aspirated for cytology 
or histology. A prospective multicenter study demonstrated a higher diagnostic yield when a solid component was present on EUS (odds ratio 2.48; 95\% confidence interval (CI) 1.1-5.6) [11]. EUS-FNA of the cyst wall may be useful for cytological analysis, which can increase the diagnostic yield for mucinous lesions by $37 \%$ [12].

The addition of EUS-FNA to CT scan and MRI increased the overall accuracy for diagnosing PCN by 36 and 54\%, respectively [13]. However, given the suboptimal performance characteristics of cytology and carcinoembryonic antigen (CEA) as well as the very low prevalence of malignancy in PCN, it is not clear which cysts should undergo EUS with or without FNA.

Dosage of intracystic amylase, lipase, and tumor markers such as CEA can be useful to increase the yield of EUS-FNA. However, the sensitivities and specificities of biochemical analysis are highly variable in the literature, making interpretation difficult. A pooled analysis of 12 studies (450 patients) demonstrated that amylase levels $<250 \mathrm{U} / 1$ had a $98 \%$ specificity to exclude the diagnosis of pseudocyst [14]. In a prospective, multicenter study (112 patients), a CEA cutoff of $192 \mathrm{ng} / \mathrm{ml}$ was found to be optimal for differentiating mucinous from non-mucinous cysts, providing a sensitivity of $75 \%$ and a specificity of $84 \%$. Lower CEA cutoff levels (30 ng/ml) have increased the sensitivity for identifying mucinous cysts without sacrificing specificity [3]. In one study, CEA $<5 \mathrm{ng} / \mathrm{ml}$ was seen in only 7\% of MCN and all SCN [15]. Higher CEA levels do not correlate with malignancy. Other studied tumor markers included CA 19-9, CA 125, CA 72-4, and CA 15-3, but none of these appear accurate enough to provide a definitive diagnosis [16].

The performance characteristics of EUS-FNA for distinguishing mucinous from non-mucinous pancreatic cysts by using cytology have been shown in two meta-analyses. Cytology from EUS-FNA aspirates had a pooled sensitivity of $63 \%$ (95\% CI 56-70\%) and specificity of $88 \%$ (95\% CI 83-93\%) in the first study [17] and 54\% (95\% CI 49-59\%) and 93\% (95\% CI 90-95\%) in the second, respectively [18]. In the latter, 1,438 patients from 18 studies were included, and surgical histology or clinical follow-up of at least 6 months was used as the reference standard [18].

The diagnostics of pseudocyst relies on suggestive cytological findings (macrophages, histiocytes, and neutrophils). The diagnostics of mucinous neoplasm relies on the presence of mucin, which is encountered in 35\% or more of the cases [19]. The diagnostics of SCN relies on glycogen-rich cuboidal cells; however, these are only present in approximately $10 \%$ of cases [19]. The yield of EUS-FNA for IPMN is poor since the cell density is very low and results of tumor marker and pancreatic enzyme dosages are highly variable $[20,21]$.

The diagnosis of malignancy within a cystic neoplasm can be performed by cytology with a specificity of $83-99 \%$; however, the sensitivity reported in the literature varies widely, i.e. from 25 to $88 \%$ [22].

Analysis of molecular markers in pancreatic cyst fluid might increase the limited accuracy of EUS-FNA by using cytology and chemical and/or tumor marker analysis alone. The detection of a $\mathrm{K}$-ras mutation was strongly associated with mucinous cysts; fur- thermore, a combined K-ras and allelic loss showed a specificity of $96 \%$ for malignancy [23]. However, a poor agreement between cyst fluid CEA levels and molecular analysis in diagnosing mucinous cysts was shown for the latter, with comparable sensitivities and diagnostic accuracies [24].

\section{Contrast-Enhanced Endoscopic Ultrasound}

Current evidence suggests that contrast-enhanced EUS $(\mathrm{CH}-$ EUS) is highly accurate for distinguishing non-neoplastic cysts (pseudocysts and dysontogenic cysts) from neoplastic cysts because the former do not exhibit cystic wall vascularization. During EUS, it is possible to use ultrasound contrast agents to depict blood flow in small vessels.

All published studies about $\mathrm{CH}$-EUS are retrospective but one. Only three articles addressed the issue of differential diagnosis of PCN. In the first study including 125 patients, contrast enhancement of cyst wall, septa, and nodules was observed in all PCN including mucinous cysts, cystic adenocarcinomas, SCN, and cystic neuroendocrine neoplasms. On the contrary, only $6 \%$ of non-neoplastic cystic lesions (pseudocysts and dysontogenic cysts) exhibit these features [25]. In another study in 76 patients with PCN, the vast majority of serous and mucinous cysts was hyperenhanced during $\mathrm{CH}$-EUS without significant difference (86 vs. $89 \%$ ). $90 \%$ of pseudocysts showed hypoenhancement ( $\mathrm{p}=0.000004$ vs. serous cysts and $\mathrm{p}=0.000005$ vs. mucinous cysts). $\mathrm{CH}$-EUS did not add any advantage to EUS alone for differentiating mucinous from non-mucinous cysts when the presence of mural nodules was considered as a sign of mucinous cysts (sensitivity 79 vs. $85 \%$; specificity 96 vs. $46 \%$ ) [26].

$\mathrm{CH}$-EUS might also be useful for distinguishing mural epithelial nodules from mucinous clots. In 17 patients with mural nodules in BD-IPMN referred for surgery, CH-EUS depicted vascularity in all pathologically confirmed nodules and in one case with mucous clots (sensitivity $100 \%$, specificity $80 \%$ ) [27]. Comparable results were confirmed in later studies. In one study by Fusaroli et al. [26], all hyperenhanced solid components during $\mathrm{CH}$-EUS were malignant whereas non-enhanced ones were either mucous clots or internal debris.

\section{Needle-Based Confocal Laser Endomicroscopy}

Needle-based confocal laser endomicroscopy (nCLE) is a new endoscopic modality allowing to image a target tissue at a subcellular level of resolution, providing real-time in-vivo optical biopsy. Images with very high spatial resolution and magnification of the focal plane examined within the tissue are thus produced. Intravenous injection of a fluorescent dye, most commonly sodium fluorescein, is required. Fluorescein acts as a contrast agent highlighting blood vessels and tissue architecture. The CLE technology is incorporated into a miniprobe of $0.85 \mathrm{~mm}$ (AQ-Flex) that can be passed through a 19-gauge EUS-FNA. This probe is provided with 
10,000 optical fibers and has a field of view of $325 \mu \mathrm{m}$, a lateral resolution of $3.5 \mu \mathrm{m}$, and a confocal depth of 40-70 $\mu \mathrm{m}$ [28]. EUS is advanced to the area of interest, and a single pass with the preloaded 19-gauge EUS-FNA is performed. The needle is advanced under EUS guidance until the needle tip contacts with the cyst wall. nCLE imaging begins after injection of fluorescein.

Most of the published studies focused on the feasibility, safety, and performance in differentiating mucinous from non-mucinous cysts or in the differential diagnosis among the several types of PCN. It is proven that nCLE is feasible during EUS-FNA and allows in-vivo diagnosis of PCN with high accuracy [29-32]. nCLE criteria have been defined for IPMN, MCN, SCN, pseudocysts, and cystic neuroendocrine tumors with proven histopathological correlation. A specificity of $100 \%$ has been shown for benign SCN and for mucinous lesions. The superficial vascular network is exclusive to SCN, allowing a diagnosis with high confidence while preventing from unnecessary surgical resection [33]. The sensitivity of this approach is also high, but epithelium denudation may account for the lack of visualization of such vascular patterns in olygocystic SCN. The specificity is also excellent for mucinous lesions. However, the distinction between IPMN and MCN is more difficult. When bleeding or inflammation occurs in an $\mathrm{MCN}$, the distinction between MCN and a pseudocyst might also prove difficult. Two inter- and intraobserver studies have confirmed nCLE reproducibility not only among experts in nCLE but also among nCLE-naive observers. The major concern is the risk of acute pancreatitis (average rate $2.7 \%$, which is comparable with that of the conventional EUS-FNA procedure) [34]. No study reported adverse events related to the injection of fluorescein, but severe allergic reactions are possible, although very uncommon. The future will show whether nCLE is useful and in which cases. As for EUS, nCLE should only be used in difficult cases when imaging workup including classical EUS failed to reach a definitive diagnosis.

\section{Adverse Events from EUS}

In a systematic review, adverse events specific to EUS-FNA of PCN occurred in $2.7 \%$ of 909 patients (5\% in prospective studies) $[2,35]$. No patient- or cyst-specific characteristics are associated with the development of an adverse event. The most common adverse events include abdominal pain, pancreatitis, and intracystic hemorrhage. Data regarding the use of prophylactic antibiotics in pancreatic cysts after EUS-FNA are equivocal. The current guidelines of the American Society for Gastrointestinal Endoscopy suggest the administration of antibiotics for 3-5 days after EUS-FNA of a pancreatic cystic lesion [2].

\section{Conclusions}

In conclusion, EUS is a third-line tool in the diagnosis of PCN. Clinical context as well as careful evaluation of CT scan and MRCP images by specialized radiologists are crucial in the diagnosis process. Nowadays it is inconceivable to skip these steps. In summary, EUS \pm FNA are very helpful in the most difficult cases in order to adapt the therapeutic or follow-up strategies.

\section{Acknowledgements}

The authors wish to thank Bertrand Napoléon for his help in preparing the manuscript.

\section{Disclosure Statement}

Authors declare no conflicts of interest.

\section{References}

1 Laurent L, et al: Estimation of the prevalence of intraductal papillary mucinous neoplasm of the pancreas in the French population through patients waiting for liver transplantation. United European Gastroenterol J 2017;5:499-503.

2 ASGE Standards of Practice Committee; Early DS, et al: Adverse events associated with EUS and EUS with FNA. Gastrointest Endosc 2013;77:839-843.

3 Brugge WR, et al: Diagnosis of pancreatic cystic neoplasms: a report of the cooperative pancreatic cyst study. Gastroenterology 2004;126:1330-1336.

$\checkmark 4$ Ahmad NA, et al: Interobserver agreement among endosonographers for the diagnosis of neoplastic versus non-neoplastic pancreatic cystic lesions. Gastrointest Endosc 2003;58:59-64.

5 Song $\mathrm{MH}$, et al: EUS in the evaluation of pancreatic cystic lesions. Gastrointest Endosc 2003;57:891-896.

6 Koito K, et al: Solitary cystic tumor of the pancreas: EUS-pathologic correlation. Gastrointest Endosc 1997; 45:268-276.
Cohen-Scali F, et al: Discrimination of unilocular macrocystic serous cystadenoma from pancreatic pseudocyst and mucinous cystadenoma with CT: initial observations. Radiology 2003;228:727-733.

${ }_{8} \mathrm{Kim} \mathrm{KW}$, et al: Imaging features to distinguish malignant and benign branch-duct type intraductal papillary mucinous neoplasms of the pancreas: a meta-analysis. Ann Surg 2014;259:72-81.

9 Tanaka M, et al: Revisions of international consensus Fukuoka guidelines for the management of IPMN of the pancreas. Pancreatology 2017;17:738-753.

10 Zhong N, et al: Histologic and imaging features of mural nodules in mucinous pancreatic cysts. Clin Gastroenterol Hepatol 2012;10:192-198, 198.e1-2.

11 Lim LG, et al: Factors determining diagnostic yield of endoscopic ultrasound guided fine-needle aspiration for pancreatic cystic lesions: a multicentre Asian study. Dig Dis Sci 2013;58:1751-1757.

12 Rogart JN, et al: Cyst wall puncture and aspiration during EUS-guided fine needle aspiration may increase the diagnostic yield of mucinous cysts of the pancreas. J Clin Gastroenterol 2011;45:164-169.
13 Levy MJ, et al: Endoscopic ultrasound-guided trucut biopsy of the cyst wall for diagnosing cystic pancreatic tumors. Clin Gastroenterol Hepatol 2005;3:974-979.

14 van der Waaij LA, van Dullemen HM, Porte RJ: Cyst fluid analysis in the differential diagnosis of pancreatic cystic lesions: a pooled analysis. Gastrointest Endosc 2005;62:383-389.

15 O'Toole D, et al: Macrocystic pancreatic cystadenoma: the role of EUS and cyst fluid analysis in distinguishing mucinous and serous lesions. Gastrointest Endosc 2004;59:823-829.

16 Farrell JJ, Fernandez-del Castillo C: Pancreatic cystic neoplasms: management and unanswered questions. Gastroenterology 2013;144:1303-1315.

17 Thosani N, et al: Role of EUS-FNA-based cytology in the diagnosis of mucinous pancreatic cystic lesions: a systematic review and meta-analysis. Dig Dis Sci 2010; 55:2756-2766

$55: 2756-2766$.
Thornton GD, et al: Endoscopic ultrasound guided fine needle aspiration for the diagnosis of pancreatic cystic neoplasms: a meta-analysis. Pancreatology 2013; $13: 48-57$ 
19 Frossard JL, et al: Performance of endosonographyguided fine needle aspiration and biopsy in the diagnosis of pancreatic cystic lesions. Am J Gastroenterol 2003;98:1516-1524.

20 Maire F, et al: Intraductal papillary mucinous tumors of the pancreas: the preoperative value of cytologic and histopathologic diagnosis. Gastrointest Endosc 2003; 58:701-706.

21 Maire F, et al: Intraductal papillary mucinous neoplasms of the pancreas: performance of pancreatic fluid analysis for positive diagnosis and the prediction of malignancy. Am J Gastroenterol 2008; 103:28712877.

22 ASGE Standards of Practice Committee; Muthusamy VR, et al: The role of endoscopy in the diagnosis and treatment of cystic pancreatic neoplasms. Gastrointest Endosc 2016;84:1-9.

23 Rockacy MJ, et al: Association between KRAS mutation, detected in pancreatic cyst fluid, and long-term outcomes of patients. Clin Gastroenterol Hepatol 2013;11:425-429.

24 Al-Haddad M, et al: Performance characteristics of molecular (DNA) analysis for the diagnosis of mucinous pancreatic cysts. Gastrointest Endosc 2014;79: 79-87.
5 Hocke M, et al: Pancreatic cystic lesions: the value of contrast-enhanced endoscopic ultrasound to influence the clinical pathway. Endosc Ultrasound 2014;3:1231230.

26 Fusaroli P, et al: Contrast harmonic-endoscopic ultrasound is useful to identify neoplastic features of pancreatic cysts (with videos). Pancreas 2016;45:265-268.

27 Yamashita Y, et al: Usefulness of contrast-enhanced endoscopic sonography for discriminating mural nodules from mucous clots in intraductal papillary mucinous neoplasms: a single-center prospective study. J Ultrasound Med 2013;32:61-68.

28 Tsujino T, et al: In vivo identification of pancreatic cystic neoplasms with needle-based confocal laser endomicroscopy. Best Pract Res Clin Gastroenterol 2015; 29:601-610.

29 Napoleon B, et al: A novel approach to the diagnosis of pancreatic serous cystadenoma: needle-based confocal laser endomicroscopy. Endoscopy 2015;47:26-32.

30 Napoleon B, et al: In vivo characterization of pancreatic cystic lesions by needle-based confocal laser endomicroscopy (nCLE): proposition of a comprehensive nCLE classification confirmed by an external retrospective evaluation. Surg Endosc 2016;30:26032612.
1 Kadayifci A, et al: Needle-based confocal laser endomicroscopy for evaluation of cystic neoplasms of the pancreas. Dig Dis Sci 2017;62:1346-1353.

32 Krishna SG, et al: In vivo and ex vivo confocal endomicroscopy of pancreatic cystic lesions: a prospective study. World J Gastroenterol 2017;23:3338-3348.

33 Jais B, et al: Serous cystic neoplasm of the pancreas: a multinational study of 2622 patients under the auspices of the International Association of Pancreatology and European Pancreatic Club (European Study Group on Cystic Tumors of the Pancreas). Gut 2016; 65:305-312.

34 Nakai Y, et al: Diagnosis of pancreatic cysts: EUSguided, through-the-needle confocal laser-induced endomicroscopy and cystoscopy trial: DETECT study. Gastrointest Endosc 2015;81:1204-1214.

35 Wang KX, et al: Assessment of morbidity and mortality associated with EUS-guided FNA: a systematic review. Gastrointest Endosc 2011;73:283-290. 